\title{
Blimpy: Breakthrough Listen I/O Methods for Python
}

\section{DOI: $10.21105 /$ joss. 01554}

\section{Software}

- Review ¿

- Repository ca

- Archive ra

Submitted: 28 June 2019 Published: 21 October 2019

\section{License}

Authors of papers retain copyright and release the work under a Creative Commons Attribution 4.0 International License (CC-BY).

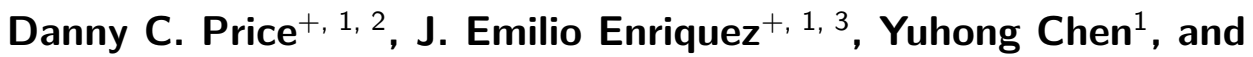 Mark Siebert ${ }^{1}$}

+ Equal contribution from both authors 1 Department of Astronomy, University of California Berkeley, Berkeley CA 94720, United States of America 2 Centre for Astrophysics \&

Supercomputing, Swinburne University of Technology, Hawthorn, VIC 3122, Australia 3 Department of Astrophysics/IMAPP, Radboud University, Nijmegen, Netherlands

\section{Summary}

The search for extraterrestrial intelligence (SETI) has historically used radio astronomy data as the main venue to search for artificial signals of extraterrestrial origin. The Breakthrough Listen program is the latest large-scale project for the search of technosignatures, and thanks to modern telescopes and instrumentation, as well as significant amounts of dedicated observing time, the program has become the largest SETI endeavour in history. This has also resulted in an unprecedented amount of publicly-available data (Lebofsky et al., 2019). Over 1 PB of data from the Breakthrough Listen program may be downloaded from seti.berkeley.edu/opendata.

The Blimpy-Breakthrough Listen I/O Methods for Python-package provides Python $2.7+/ 3.6+$ utilities for viewing and interacting with the data formats used within the Breakthrough Listen program. This includes Sigproc filterbank (.fil) and HDF5 (.h5) files that contain dynamic spectra (aka 'waterfalls'), and GUPPI raw (.raw) files that contain voltage-level data. Python methods for data extraction, calibration, and visualization are provided. A suite of command-line utilities are also available.

The waterfall data product stores an array of detected power across frequency channel (i.e. spectra) over time. These files can be several GB in size, with up to billions of channels and/or hundreds of thousands of time steps. Blimpy provides convenient methods to extract frequencies and time slices of interest-without loading the full file into memory-which are presented as Numpy arrays (van der Walt, Colbert, \& Varoquaux, 2011). Methods for manipulating lower-level voltage data products stored in the GUPPI raw format, as generated by the Green Bank Telescope, are also provided. Blimpy uses the Matplotlib library (Hunter, 2007) to provide plotting of spectra, time series, and dynamic spectra; the Astropy package for handling of astronomical coordinates (Astropy Collaboration et al., 2013; Price-Whelan et al., 2018); and, the H5py package to interact with data stored in HDF5 files (Collette, 2013). The turboSETI package, which conducts doppler acceleration searches for narrowband signals that would indicate the presence of technologically-capable life beyond Earth, uses Blimpy for file handling and diagnostic plotting.

Blimpy was designed to be used by radio astronomers, students and anyone else interested in accessing Breakthrough Listen data, whether searching for SETI signals, spectral lines, pulsars, fast radio bursts, or other astrophysical phenomena. It has already been used in a number of scientific publications (Croft et al., 2018; Enriquez et al., 2019, 2018; Enriquez et al., 2017; Gajjar et al., 2018; Price, Croft, et al., 2019; Price, Enriquez, et al., 2019). 


\section{Acknowledgements}

We thank G. Molenaar and B. Brzycki for their code contributions, along with G. Zhang, G. Hellbourg, N. Richard, M. Lebofsky, G. Foster, C. Gilbertson, and the wider Breakthrough Listen collaboration. Breakthrough Listen is managed by the Breakthrough Initiatives, sponsored by the Breakthrough Prize Foundation.

\section{References}

Astropy Collaboration, Robitaille, T. P., Tollerud, E. J., Greenfield, P., Droettboom, M., Bray, E., Aldcroft, T., et al. (2013). Astropy: A community Python package for astronomy, 558, A33. doi:10.1051/0004-6361/201322068

Collette, A. (2013). Python and hdf5. O'Reilly.

Croft, S., Siemion, A., De Boer, D., Enriquez, J. E., Foster, G., Gajjar, V., Hellbourg, G., et al. (2018). The Breakthrough Listen Search for Intelligent Life. In American astronomical society meeting abstracts \#231, American astronomical society meeting abstracts (Vol. 231, p. 231.01).

Enriquez, J. E., Siemion, A., Dana, R., Croft, S., Méndez, A., Xu, A., Deboer, D., et al. (2019). Breakthrough Listen follow-up of the reported transient signal observed at the Arecibo Telescope in the direction of Ross 128. International Journal of Astrobiology, 18, 33-35. doi:10.1017/S1473550417000465

Enriquez, J. E., Siemion, A., Foster, G., Gajjar, V., Hellbourg, G., Hickish, J., Isaacson, H., et al. (2017). The Breakthrough Listen Search for Intelligent Life: $1.11 .9 \mathrm{GHz}$ Observations of 692 Nearby Stars. ApJ, 849(2), 104. doi:10.3847/1538-4357/aa8d1b

Enriquez, J. E., Siemion, A., Lazio, T. J. W., Lebofsky, M., MacMahon, D. H. E., Park, R. S., Croft, S., et al. (2018). Breakthrough Listen Observations of 1l/ Oumuamua with the GBT. Research Notes of the American Astronomical Society, 2, 9. doi:10.3847/2515-5172/aaa6c9

Gajjar, V., Siemion, A. P. V., Price, D. C., Law, C. J., Michilli, D., Hessels, J. W. T., Chatterjee, S., et al. (2018). Highest Frequency Detection of FRB 121102 at 4-8 GHz Using the Breakthrough Listen Digital Backend at the Green Bank Telescope, 863, 2. doi:10.3847/ 1538-4357/aad005

Hunter, J. D. (2007). Matplotlib: A 2D graphics environment. Computing in Science \& Engineering, 9(3), 90-95. doi:10.1109/MCSE.2007.55

Lebofsky, M., Croft, S., Siemion, A. P. V., Price, D. C., Enriquez, J. E., Isaacson, H., MacMahon, D. H. E., et al. (2019). The Breakthrough Listen Search for Intelligent Life: Public Data, Formats, Reduction and Archiving. arXiv e-prints, arXiv:1906.07391. Retrieved from http://arxiv.org/abs/1906.07391

Price, D. C., Croft, S., DeBoer, D., Drew, J., Enriquez, J. E., Foster, G., Gajjar, V., et al. (2019). Breakthrough Listen Observations of Asteroid (514107) 2015 BZ $_{509}$ with the Parkes Radio Telescope. Research Notes of the American Astronomical Society, 3, 19. doi:10.3847/ 2515-5172/ab010b

Price, D. C., Enriquez, J. E., Brzycki, B., Croft, S., Czech, D., DeBoer, D., DeMarines, J., et al. (2019). The Breakthrough Listen Search for Intelligent Life: Observations of 1327 Nearby Stars over 1.10-3.45 GHz. arXiv e-prints, arXiv:1906.07750. Retrieved from http://arxiv.org/abs/1906.07750 
Price-Whelan, A. M., Sipöcz, B. M., Günther, H. M., Lim, P. L., Crawford, S. M., Conseil, S., Shupe, D. L., et al. (2018). The Astropy Project: Building an Open-science Project and Status of the v2.0 Core Package, 156, 123. doi:10.3847/1538-3881/aabc4f

van der Walt, S., Colbert, S. C., \& Varoquaux, G. (2011). The NumPy Array: A Structure for Efficient Numerical Computation. Computing in Science and Engineering, 13(2), 22-30. doi:10.1109/MCSE.2011.37 